\title{
Dirac Fermionic Dark Matter Confronting the Latest Data
}

\section{Gwo-Guang Wong*}

Department of Physics and Chung Yuan Center for High Energy Physics, Chung Yuan Christian University,

Chung-Li, Taiwan 320, Republic of China E-mail:

\section{Chun-Khiang Chua}

Department of Physics and Chung Yuan Center for High Energy Physics,

Chung Yuan Christian University,

Chung-Li, Taiwan 320, Republic of China E-mail: Ckchuadcycu.edw.tw

\begin{abstract}
It is well known that the Dirac fermionic dark matter (DM) is seriously constrained by the spinindependent (SI) experiments of DM-nucleus elastic scattering. To evade the vector interaction of DM with the $Z$ boson, we take the quantum numbers of the Dirac DM to be $I \neq 0, I_{3}=Y=0$. The effective couplings from loop contribution should be considered. We find that there is a cancellation in one-loop diagrams, which largely reduces the cross section and makes the Dirac DM viable in the direct search. Besides, for a generic isospin $I$, we survey the Dirac DM mass constrained by the recent results of PandaX-II, XENON1T and LUX experiments in the direct search, the observed DM relic density, and the H.E.S.S and Fermi-LAT astrophysical observations in the indirect search. With the Sommerfeld enhancement effect, we find that the H.E.S.S. data and the observed DM relic density become serious constraints on the DM mass. The $I=1$ case is ruled out and Dirac DM masses are forced to be within the ranges of a few to few tens $\mathrm{TeV}$ for $2 \leqslant I \leqslant 4$ and few tens to few hundreds TeV for $I \geqslant 5$.
\end{abstract}

The European Physical Society Conference on High Energy Physics

5-12 July 2017

Venice, Italy

${ }^{*}$ Speaker. 


\section{Introduction}

One of the most promising DM candidate is so-called the weakly interacting massive particles (WIMPs) which are non-luminous and non-baryonic cold DM (CDM) matter. The DM particles are assumed to be created thermally during the big bang, and froze out of thermal equilibrium escaping the Boltzmann suppression in the early Universe [U] and the recent measured value of DM relic density is $\Omega_{\mathrm{obs}} h^{2}=0.1186 \pm 0.0020$ []. So for, there are three complementary searching strategies to detect the DM particles in experiments including the direct detection of DM-nucleus scattering in underground laboratories, the indirect detection of DM annihilation processes in astrophysical observation and the DM direct production at colliders. The null results of finding the DM from direct search of PandaX-II [B], XENON1T [ [ 9 ], LUX [5] experiments, and from the indirect search of H.E.S.S [可] and Fermi-LAT [ $\square]$ astrophysical observations put the related upper limits on spin-independent (SI), spin-dependent (SD) DM-nucleus scattering cross sections and the velocity averaged DM annihilation cross sections respectively.

In this report, we work on a Dirac fermionic dark matter model by adding a Dirac fermionic multiplet $\chi$ with arbitrary $I$ and $Y$ on standard model (SM), and the Lagrangian can be written as [8]

$$
\begin{aligned}
\mathscr{L} & =\mathscr{L}_{S M}+\bar{\chi}\left(i \gamma^{\mu} D_{\mu}-m_{\chi}\right) \chi \\
& =\mathscr{L}_{S M}+\bar{\chi}^{j} \gamma^{\mu}\left(i \partial_{\mu}-g W_{\mu}^{a} T_{j k}^{a}+g^{\prime} B_{\mu} Y_{j} \delta_{j k}\right) \chi^{k}-m_{\chi} \bar{\chi}^{i} \chi^{i} \\
& \supset \frac{g}{\cos \theta_{W}} T^{3} \bar{\chi}^{0} \gamma^{\mu} \chi^{0} Z_{\mu} .
\end{aligned}
$$

where $W_{\mu}^{a}$ and $B_{\mu}$ are the known electroweak $\mathrm{SU}(2)_{\mathrm{L}}$ and $\mathrm{U}(1)$ gauge fields, respectively, and $\theta_{w}$ and $g$ is the weak mixing angle and weak coupling, repectively. In this model, the SM is minimally extended so that the DM can only interact with the SM gauge bosons. Besides, there is only one free parameter, the DM mass $m_{\chi}$ that makes the model much simpler. As in most DM models, we may assign DM to be $Z_{2}$-odd and SM particles $Z_{2}$-even to maintain the stability of DM. In this way, the DM do not interact with SM Higgs and fermions at tree level. From Eq. (II. $)$, we assign the DM particle with quantum numbers $I \neq 0, I_{3}=Y=0$ to avoid the vector interaction of DM with the $Z$ boson at tree level so that the spin-independent (SI) cross section $\sigma^{S I}$ of MD-nucleus elastic scattering is vanishing at tree level.
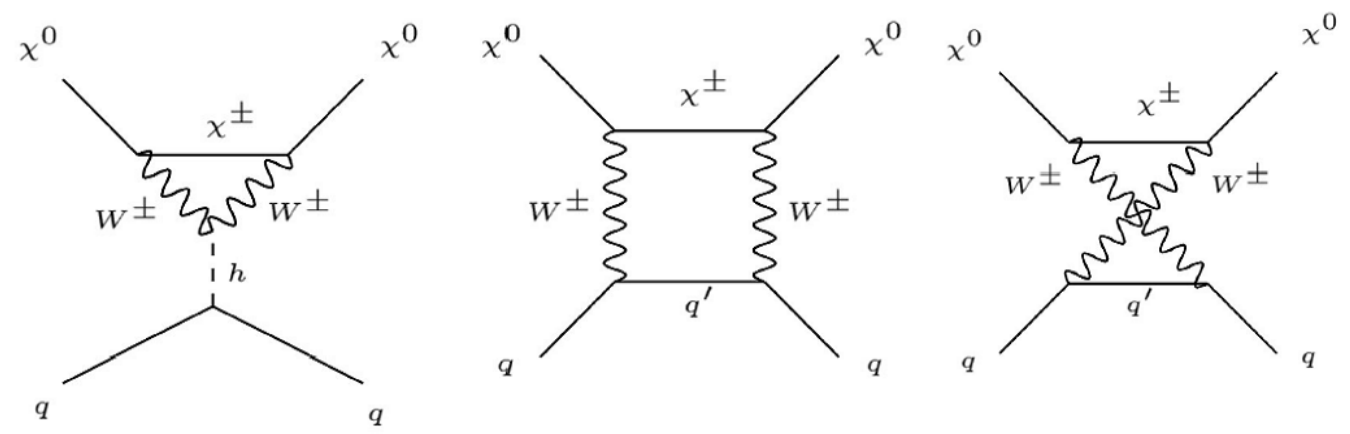

Figure 1: Non-vanishing one-loop diagrams for the effective interaction of Dirac DM with quarks 


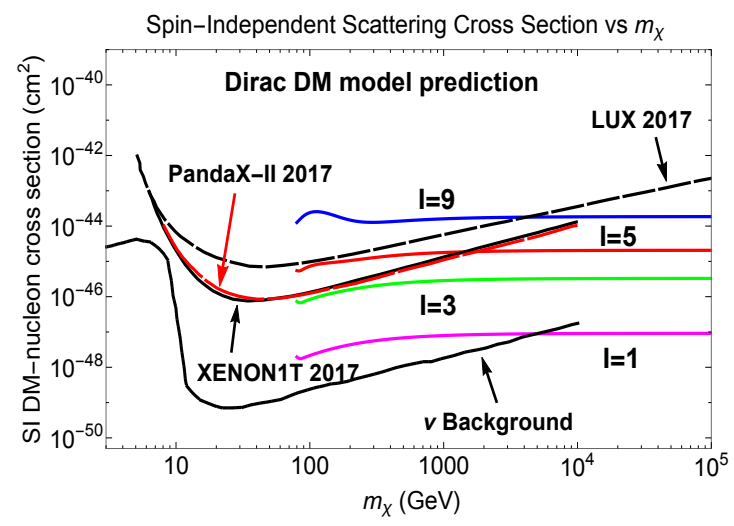

(a)

Figure 2: Dirac DM model prediction for SI cross section of DM scattering off the nuclei ${ }^{129,131} \mathrm{Xe}$ in the direct search.

\section{Dirac fermionic dark matter in the direct search}

In spite of $\sigma^{S I}=0$ at tree level, we need to consider the non-vanishing one-loop diagrams for the effective interaction of Dirac DM with quarks shown in Fig. $\mathrm{m}$. We find that there exists a cancellation so that the large component $\left(\sim 1 / M_{W}^{2}\right)$ of the effective vector-vector (VV) coupling is vanishing for all quark flavors (see [Q]]).

In Fig. $\square$, we show the plot of $\sigma^{S I}$ versus $m_{\chi}$. The prediction values sitting simultaneously below the upper limits of PandaX-II and XENON1T SI-experiments [3], 团] are allowed. We also show the upper limit of LUX SI-experiment, especially for DM mass greater than $10 \mathrm{TeV}$. We survey the DM mass from the W-boson mass to $100 \mathrm{TeV}$. For $1 \leq I \leq 3$, all $\mathrm{DM}$ mass in this region are allowed in principle. However, for $I=1$ we can not distinguish the DM event from neutrino event when $m_{\chi}>5.2 \mathrm{TeV}$ below the curve of the neutrino background. For $I>3$, we find that PandaX-II provides a lower mass bound with $m_{\chi}=0.67,1.93,3.51,6.75$ and $10.00 \mathrm{GeV}$ for $I=4,5,6,7$ and 8 , respectively. Hence the cancellation in the large component $\left(\sim 1 / M_{W}^{2}\right)$ of $\mathrm{VV}$ interaction, largely reduces the cross section of DM-nucleus elastic scattering and makes the Dirac DM model viable in the direct search.

\section{Dirac Fermionic Dark Matter in the Indirect Search}

Let us now consider the DM annihilation processes. The DM particles became non-relativistic when they froze out of thermal equilibrium in the early universe. Hence we need to take into account the Sommerfeld enhancement effect [ए]]. Analytic formulas of the Sommerfeld enhancement factors for arbitrary $I$ are obtained using the SU(2) symmetric limit and the Hulthén approximation [Q].

Figs. B(a) and B(b) show the plots of $\left\langle\sigma\left(\chi^{0} \bar{\chi}^{0} \rightarrow W^{+} W^{-}\right) v\right\rangle$ versus $m_{\chi}$ without and with considering the Sommerfeld enhancement effect for $I=1,3,5,7$ and 9. In each plot, we see that the low mass region with $m_{\chi} \leqslant 0.55 \mathrm{TeV}$ are ruled out by the Fermi-LAT constraint and the H.E.S.S data is use to constrain the mass region with $0.55<m_{\chi} \leqslant 70 \mathrm{TeV}$. The Sommerfeld enhancement 


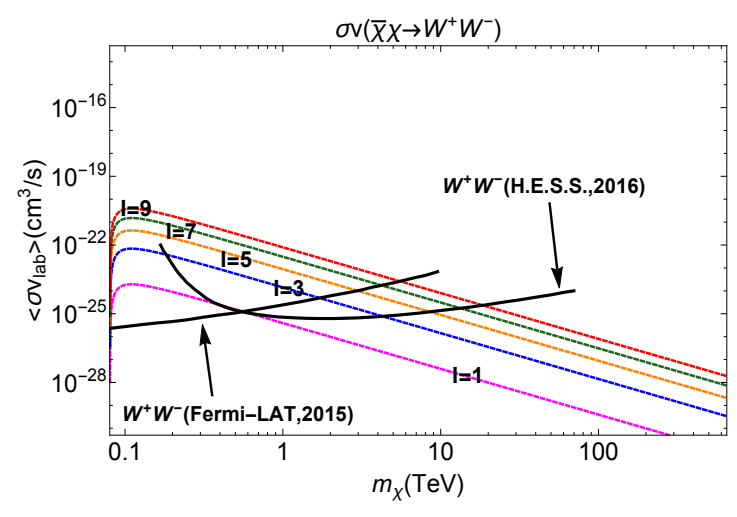

(a) without Sommerfeld enhancement effect

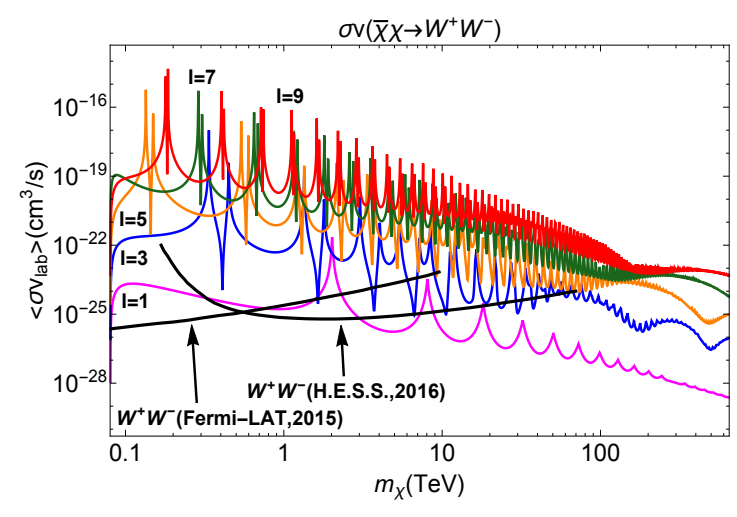

(b) with Sommerfeld enhancement effect

Figure 3: Predicted velocity averaged cross sections of galactic DM annihilation to $W^{+} W^{-}$ processe for $I=1,3,5,7$ and 9 cases.

increases the cross sections by 1 to 4 order of magnitude and, hence, the lower limits on the Dirac DM masses are in principle shifted to larger values.

Fig. $\mathbb{t}$ shows the predicted relic density using $x_{f}=24$ for different $I=1,2,4,5,7$. The solid (dashed) lines denote the results with (without) the Sommerfeld enhancement effect. The shadow area shows the observed relic density $\Omega_{\mathrm{obs}} h^{2}=0.1186 \pm 0.0020$ [[]]. This observed relic density provides us the upper mass bounds for DM particles with different isospin $I$.

\section{Conclusion}

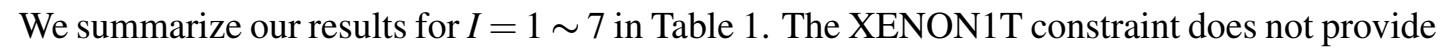
lower limits on DM masses for $I \leqslant 3$. For the larger $I$ case, the lower mass limit constrained from

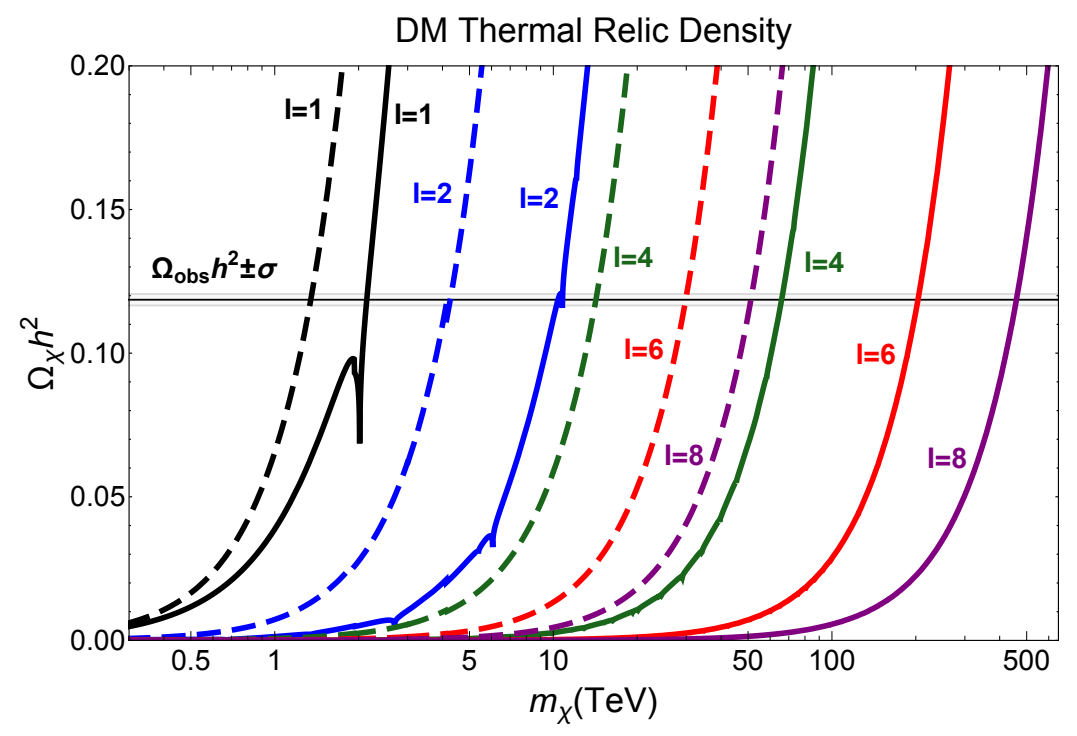

Figure 4: Predicted thermal relic density for $I=1,2,4,5,7$. 
Table 1: The lower limits $m_{\chi}^{L L}$ obtained from direc and indirect search, the upper limits $m_{\chi}^{U L}$ obtained from the observed relic density are shown. The upper values (lower values within the parentheses) for indirect $m_{\chi}^{L L}$ and relic $m_{\chi}^{U L}$ denote the results with (without) considering the Sommerfeld effect. Dirac DM mass are given in the unit of TeV.

\begin{tabular}{|c|c|c|c|c|c|c|c|}
\hline$I$ & 1 & 2 & 3 & 4 & 5 & 6 & 7 \\
\hline Direct $m_{\chi}^{L L}$ & - & - & - & 0.67 & 1.93 & 3.51 & 6.75 \\
\hline \multirow{2}{*}{ Indirect $m_{\chi}^{L L}$} & 3.00 & 1.09 & 39.12 & 41.16 & 67.33 & $>70$ & $>70$ \\
& $(0.60)$ & $(2.38)$ & $(4.29)$ & $(6.38)$ & $(8.55)$ & $(10.86)$ & $(13.34)$ \\
\hline \multirow{2}{*}{ Relic $m_{\chi}^{U L}$} & 2.15 & 10.58 & 30.30 & 66.54 & 123.20 & 205.23 & 316.94 \\
& $(1.36)$ & $(4.30)$ & $(8.60)$ & $(14.34)$ & $(21.51)$ & $(30.11)$ & $(41.14)$ \\
\hline
\end{tabular}

the indirect search is more stringent than that from the direct search. The Fermi-LAT and H.E.S.S. upper limits provide lower bounds on $m_{\chi}$, while the relic density provides upper bounds. Without considering the Sommerfeld effect, all cases (with different isospin I) are allowed, while after turning on the Sommerfeld effect, the $I=1$ case is ruled out and the Dirac mass is forced to be within the ranges of a few to few tens TeV for $2 \leqslant I \leqslant 4$ and few tens to few hundreds $\mathrm{TeV}$ for $I \geqslant 5$.

\section{Acknowledgments}

This research was supported by the Ministry of Science and Technology of R.O.C. under Grant Nos. 105-2811-M-033-007 and in part by 103-2112-M-033-002-MY3 and 106-2112-M-033 -004 -MY3.

\section{References}

[1] G. Jungman, M. Kamionkowski and K. Griest, Phys. Rept. 267, 195 (1996) [hep-ph/9506380].

[2] K. A. Olive et al. [Particle Data Group Collaboration], Chin. Phys. C 38, 090001 (2014).

[3] X. Cui et al. [PandaX-II Collaboration], "Dark Matter Results From 54-Ton-Day Exposure of PandaX-II Experiment," arXiv:1708.06917 [astro-ph.CO].

[4] E. Aprile et al. [XENON Collaboration], arXiv:1705.06655 [astro-ph.CO].

[5] D. S. Akerib et al. [LUX Collaboration], Phys. Rev. Lett. 118, no. 2, 021303 (2017) [arXiv:1608.07648 [astro-ph.CO]].

[6] H. Abdallah et al. [H.E.S.S. Collaboration], Phys. Rev. Lett. 117, no. 11, 111301 (2016) [arXiv:1607.08142 [astro-ph.HE]].

[7] M. Ackermann et al. [Fermi-LAT Collaboration], Phys. Rev. Lett. 115, no. 23, 231301 (2015) [arXiv:1503.02641 [astro-ph.HE]].

[8] M. Cirelli, N. Fornengo and A. Strumia, Nucl. Phys. B 753, 178 (2006) [hep-ph/0512090].

[9] C. K. Chua and G. G. Wong, [arXiv:1708.08624 [hep-ph]].

[10] J. Hisano, S. Matsumoto and M. M. Nojiri, Phys. Rev. Lett. 92, 031303 (2004) [hep-ph/0307216]. 